\title{
Protokoll Mitgliederversammlung der DEGUM Freitag, 28. Oktober 2016, 17:30 Uhr
}

\author{
40. Dreiländertreffen in Leipzig, Congress Center Leipzig, Raum MZF3
}

Teilnehmer: 143

\section{Eröffnung}

Der Neupräsident PD Dr. med. K.-S. Heling begrüßt die Teilnehmer der Mitgliederversammlung.

\section{Ad TOP 1: Annahme der}

\section{Tagesordnung}

Die Einladung mit Tagesordnung wurde satzungsgemäß in der Zeitschrift „Ultraschall in der Medizin" sowie auf der Homepage der DEGUM veröffentlicht. K.-S. Heling stellt den Antrag, die Tagesordnung wie veröffentlicht zu bestätigen:

- TOP 1: Annahme der Tagesordnung

- TOP 2: Annahme des Protokolls der Mitgliederversammlung am 25.09.15 bei dem 39. Dreiländertreffen in Davos

- TOP 3: Bericht des Vorstandes

- TOP 4: Bericht des Schatzmeisters

- TOP 5: Entlastung des Vorstandes

- TOP 6: Aufnahme neuer Mitglieder

- TOP 7: Ehrungen

- Preise (Wissenschaft und Promotion)

- Ehrenmitgliedschaften

- TOP 8: Wahlen

- TOP 9: Kurzberichte aus den Sektionen und Arbeitskreisen

- TOP 10: Verschiedenes

Die Tagesordnung wird einstimmig angenommen.

\section{Ad TOP 2: Annahme des Protokolls der Mitglieder- versammlung am 25.09.2015 bei dem 39. Dreiländertreffen in Davos}

Das Protokoll wird einstimmig angenommen.

\section{Ad TOP 3: Bericht des Vorstandes}

K.-S. Heling erläutert die reduzierte Präsenz des Vorstandes:

D. Becker kann aus klinikinternen Gründen nicht am Kongress teilnehmen, W. Mann ist terminlich verhindert und $P$. Jecker ist durch eine familiäre Trauerfeier verhindert, an der Mitgliederversammlung teilzunehmen. $\mathrm{Er}$ weist darauf hin, dass daher in der Vorstandssitzung vom 26.10.16 beschlossen wurde, dass der Bericht des Vorstandes durch den Neupräsidenten präsentiert wird.

K.-S. Heling gibt einen Überblick über das vergangene Jahr - folgende Punkte werden besonders herausgehoben:

Die Vertragsverhandlungen mit dem Thieme-Verlag über die Zeitschrift, Ultraschall in der Medizin“ wurden bereits in der Mitgliederversammlung im letzten Jahr in Davos ausführlich präsentiert. Der Preis ist für die nächsten fünf Jahre stabil, das Format wird beibehalten. Der Impact-Factor ist mit 4.434 weiterhin sehr hoch. Die Zeitschrift nimmt unter 124 Zeitschriften in der Kategorie ,Radiology, Nuclear Medicine \& Medical Imaging den 13. Platz ein und in der Kategorie ,Acoustics“ von 32 Zeitschriften
Platz 2. Für die Bezugsvariante Online Only haben sich ca. 700 Mitglieder entschieden.

Die Novellierung der Ultraschallvereinbarung stand in diesem Jahr als Thema auf der Liste. Vonseiten der DEGUM entstand der Wunsch nach Flexibilisierung des Kurssystems, weg vom starren Prinzip des Grund-, Aufbau- und Abschlusskurses hin zu einem modularen System, das von den Sektionen Innere Medizin, Radiologie und Chirurgie bevorzugt wird. So kann es gelingen, schneller auf technische Neurungen etc. einzugehen. Die Sektion Gynäkologie \& Geburtshilfe hingegen bleibt weiterhin bei dem bisherigen System, um die Anerkennung der KV zu erreichen.

Die DEGUM wurde von der KBV in vielen Bereichen in die Diskussion miteinbezogen ein Ergebnis ist, dass der Grundkurs Abdomen jetzt auch über drei Tage gestaltet werden kann, allerdings bleibt zu beachten, dass der Interdisziplinäre Grundkurs inklusive Schilddrüse weiterhin an vier Tagen und 30 Stunden durchgeführt werden muss. Die neue Fassung der Ultraschallvereinbarung ist nach einem sehr zügigen letzten Durchlauf bereits verabschiedet und seit dem 01.10.16 in Kraft. Auf der Homepage der DEGUM ist die neue Version sowie alle Änderungen im Überblick einzusehen.

Die Arbeit in der Geschäftsstelle mit beiden Standorten Bonn und Berlin wird von den insgesamt 6 Mitarbeiterinnen in hervorragender Weise geleistet. K.-S. Heling bedankt sich sehr herzlich bei allen für die exzellente Arbeit. Langfristig werden beide Standorte in Berlin zusammengelegt werden. Dafür werden neue Räumlichkeiten 
gesucht, die den Platzbedarf für e. V. und Akademie abdecken.

Die Entwicklung einer neuen Mitglieder-Datenbank ist weit vorangeschritten und wird in 2017 freigeschaltet, was die Arbeit insgesamt sehr erleichtern wird.

Herr Rechtsanwalt Schumacher hat im März 2016 seine Tätigkeit für die DEGUM eingestellt. Über viele Jahre hat er die DEGUM in allen rechtlichen Fragestellungen hervorragend beraten und ihm sei an dieser Stelle dafür sehr herzlich gedankt! Die Rechtsberatung wird seither von Anwälten der Kanzlei BakerTillyRoelfs betreut, die Gesellschaft, die auch die steuerliche Beratung der DEGUM übernommen hat.

In der Ultraschall-Akademie hat Frau Dr. Jagota zu Ende April 2016 ihre Arbeit beendet und als Nachfolgerin hat sich nach einem Auswahlverfahren ihre bisherige Stellvertreterin Frau Beck bereits sehr gut in ihren neuen Aufgabenbereich eingearbeitet.

Die Finanzierung der zukünftigen deutschen Dreiländertreffen ist neu überdacht worden. Bisher wurden für die Kongresse Rücklagen gebildet, die eine stattliche Größenordnung erreicht hatten. Für diesen Kongress sind alle Bereiche bereits geprüft worden, um ein neutrales Ergebnis zu erreichen.

Für die nächsten DLTs in Deutschland 2019 und 2022 hat sich der Vorstand entschieden, einen Kongressort zu wählen, um so bessere Konditionen zu erhalten. Nach intensiver Beratung wurde als Kongressort für 2019 und 2022 Mainz bestimmt. Bei der Frage des Kongresspräsidiums mit einem doch erheblichen Aufgabengebiet hat sich der Vorstand entschieden, zwei Kongresspräsidenten zu wählen, die möglichst aus den großen Sektionen Innere Medizin und Gynäkologie \& Geburtshilfe kommen sollen, um möglichst viele Fachbereiche so abzudecken. Die Ernennung wird in der Sitzung des Erweiterten Vorstands im Januar 2017 erfolgen.

Die Wissenschafts- und Ausbildungsförderung in der DEGUM ist in den vergangenen Jahren aufgestockt worden. Alle eingereichten Anträge für die Wissenschaftsförderung werden nach einem festgelegten Verfahren von externen Gutachtern bewertet - die Anträge sollen die Voraussetzun- gen, die auf der Homepage der DEGUM veröffentlich sind, erfüllen. Eine parallele Beantragung bei zwei Gesellschaften ist ausgeschlossen.

Konzepte für Förderung der studentischen wie auch ärztlichen Aus- und Weiterbildung sollen in den nächsten Jahren weiter entwickelt werden.

In der letzten Zeitschrift ,Ultraschall in der Medizin' hat Herr Blank einen Bericht über einen Einsatz in Nepal geschildert - ebenfalls mitfinanziert durch die DEGUM, beantragt nach dem Leitfaden für Ausbildungsförderung im Ausland.

K.-S. Heling bedankt sich abschließend bei allen Mitgliedern des Vorstands, besonders bei D. Becker und W. Mann sowie bei A. Hagendorff als Kongresspräsident.

Im Anschluss an den Bericht wird durch ein Mitglied des Auditoriums darauf hingewiesen, dass nicht nur die beiden großen Sektionen Innere und Gynäkologie inhaltlich die zukünftigen Kongresse bestimmen sollen - er vermisste eine ausreichende Beachtung der Pädiatrie. Ebenfalls sollte stärker beachtet werden, dass wichtige Sitzungen nicht parallel angeboten werden. Zu begrüßen wären zukünftig geringere Kongressgebühren.

\section{Ad TOP 4: Bericht des Schatzmeisters}

P. Kozlowski präsentiert den Jahresabschluss 2015, den Zwischenbericht vom 30.06.16 sowie die Prognose des Haushaltes zum 31.12.16. Die Kalkulationen des Haushaltes 2016 erfolgen auf Basis von 9740 Mitgliedern und eines Beitrages in Höhe von $100 €$.

Er erläutert ausführlich die Gewinn- und Verlustrechnung unter Betonung der Personal-, Reise-, Veranstaltungs- und Raumkosten und unterstreicht, dass die gesetzten Ziele erreicht wurden.

Die Vorschläge für die Rückstellung der gebundenen Rücklagen (insgesamt $137225 €$ ) und die freien Rücklagen (418 515€) werden vorgestellt und abschließend die Trendentwicklungen sowie die Bilanz erläutert.
Die bereits im letzten Jahr vorgestellte Einsparung von 10,00€/Jahr/Mitglied bei Online Only-Bezug der Zeitschrift erzielt bei 700 Mitgliedern, die diese Variante gewählt haben, eine Einsparung von $7000 €$, die ebenfalls in der Wissenschafts- und Ausbildungsförderung eingesetzt werden sollen. Diejenigen Mitglieder, die diese 10,00€ gern zurück hätten, mögen sich bitte in der Geschäftsstelle melden.

Der Jahresabschluss 2015 sowie die Vorschläge für die Rückstellungen werden von der Mitgliederversammlung mit einer Gegenstimme angenommen.

\section{Ad TOP 5: Entlastung des Vorstandes}

Prof. K.-V. Jenderka beantragt die Entlastung des Vorstandes.

Diesem Antrag wird mit 4 Enthaltungen zugestimmt.

\section{Ad TOP 6: Aufnahme neuer Mitglieder}

Seit der letzten Mitgliederversammlung in Davos haben 619 Personen einen schriftlichen Antrag auf Mitgliedschaft gestellt. Der Vorstand hat diesen Anträgen in seinen Sitzungen zugestimmt.

Die Namen dieser durch den Vorstand aufgenommenen Neumitglieder sind den Mitgliedern der DEGUM durch Auslage am Stand der DEGUM bekannt gegeben worden. Widerspruch gegen die Aufnahme eines oder mehrerer dieser Neumitglieder wurde weder vor noch auf der Mitgliederversammlung erhoben.

Die DEGUM hat aktuell 10246 Mitglieder, davon 9957 ordentliche.

Es sind 2797 Mitglieder mit der Stufe 1 zertifiziert, 1592 mit der Stufe 2, 457 sind mit der Stufe 3 zertifiziert und 711 haben den Kursleiterstatus der Stufe 2 oder 3.

Insgesamt wurden in 2016 bisher ca. 900 Anträge zur Zertifizierung eingereicht. 
An dieser Stelle wird allen Gutachtern sowie den Mitarbeiterinnen der Geschäftsstelle für ihre Arbeit herzlich gedankt.

\section{Ad TOP 7: Ehrungen}

\section{Preise}

Für die diesjährigen Preise wurden 4 Arbeiten für den Promotionspreis und 3 Arbeiten für den Wissenschaftspreis eingereicht.

\section{Preisträger}

\section{Promotionspreis 2016}

Frau Dr. med. Claudia Schröder, Hamburg, erhält den Promotionspreis 2016 für die Arbeit:

„Real-Time Elastographie und Kontrastmittelsonographie in der Bewertung skrotaler Raumforderungen: eine vergleichende, prospektive Studie“

K.S. Heling überreicht Frau Dr. Schröder nach der Präsentation der Arbeit die Urkunde inklusive Scheck.

\section{Wissenschaftspreis 2016}

Der Wissenschaftspreis 2016 wird geteilt:

Frau Dr. med. Gwendolin Manegold-Brauer, Basel, erhält den DEGUM-Wissenschaftspreis 2016 für die Arbeit:

„Die Rolle des 11 - 14 Schwangerschaftswochen (SSW) Ultraschalls in der Ära nichtinvasiver Pränataltests"

PD Dr. med. Thomas Karlas, Leipzig, erhält den DEGUM-Wissenschaftspreis 2016 für die Arbeit:

„Analyse der Gewebeelastizität bei Lebererkrankungen: Etablierung und Anwendung Ultraschall-basierter Verfahren“

Nach den Präsentationen der Arbeiten überreicht K.-S. Heling jeweils Urkunde und Scheck.

\section{Ehrenmedaille}

Verleihung der Ehrenmedaille an Prof. Dr. med. W. Wermke

Im Rahmen der Ehren-Lecture am Vortag wurde Herr Prof. Wolfram Wermke für seine außerordentlichen Verdienste um den Ultraschall die Ehrenmedaille der DEGUM verliehen - Laudator war PD Dr. med. Karlheinz Seitz, Sigmaringen.

\section{Ehrenmitgliedschaften}

Neuantrag Prof. Dr. Ch. Arning

Prof Dr. med. M. Nedelmann, stellvertretender Vorsitzender der Sektion Neurologie, beantragt die Ehrenmitgliedschaft für Prof. Dr. med. Christian Arning. Der Antrag wurde satzungsgemäß in den Sitzungen des Vorstandes wie des Erweiterten Vorstandes einstimmig angenommen.

\section{Die Mitgliederversammlung stimmt diesem Antrag einstimmig zu.}

Damit wird die Ehrenmitgliedschaft für Prof. Arning anlässlich des nächsten Dreiländertreffens in Linz verliehen.

Verleihung der Ehrenmitgliedschaft an Prof. Dr. med. K.-H. Deeg

Die Ehrenmitgliedschaft der DEGUM wird Herrn Prof. Dr. med. Karl-Heinz Deeg verliehen. Dem Antrag war anlässlich der Mitgliederversammlung 2015 in Davos zugestimmt worden. Die Laudatio hält PD Dr. med. Udo Vester.

\section{Ad TOP 8: Wahlen}

\section{Wahl des Wahlleiters}

Als Wahlleiter wird Dr. med. Bernd FrentzelBeyme vorgeschlagen.

Diesem Vorschlag wird einstimmig zugestimmt.

Satzungsgemäß wechselt der Präsident Prof. Dr. med. Dirk. Becker in die Position des Vizepräsidenten, der Vizepräsident Prof. Dr. med. Dr. h. c. Wolf Mann verlässt den Vorstand und der Neupräsident PD Dr. med. Kai-Sven Heling wechselt in die Position des Präsidenten.

Zu wählen sind die Positionen des Neupräsidenten, des Sekretärs, des Schatzmeisters sowie der beiden Beisitzer.

Der Wahlleiter schlägt vor, die Wahlen in offener Abstimmung vorzunehmen - dieser Vorschlag wird von der Mitgliederversammlung ohne Gegenstimme angenommen.

\section{Neupräsident}

Um die Position des Neupräsidenten bewirbt sich Prof. Dr. med. Peter Jecker, der bisher die Position des Sekretärs innehatte. Da er bei dieser Abstimmung nicht anwesend sein kann, hat er schriftlich seine Bereitschaft zur Kandidatur erklärt sowie bei positivem Wahlergebnis, seine Kandidatur auch anzunehmen.

Keine weiteren Vorschläge.

Ergebnis: Prof. Dr. med. Peter Jecker wird in offener Abstimmung einstimmig gewählt.

\section{Sekretär}

Um die Position des Sekretärs bewirbt sich Prof. Dr. med. Andreas Hagendorff. Er stellt sich als aktueller Kongresspräsident der Mitgliederversammlung kurz vor.

Keine weiteren Vorschläge.

Ergebnis: Prof. Dr. med. Andreas Hagendorff wird in offener Abstimmung mit einer Enthaltung gewählt.

\section{Schatzmeister:}

Um die Position des Schatzmeisters bewirbt sich der bisherige Schatzmeister, Prof. Dr. med. Peter Kozlowski. Keine weiteren Vorschläge.

Ergebnis: Prof. Dr. med. Peter Kozlowski wird in offener Abstimmung mit einer Enthaltung gewählt.

\section{Beisitzer}

Prof. Dr. med. Markus Hahn erklärt seine Bereitschaft zur Kandidatur als Beisitzer kein weiterer Vorschlag

Ergebnis: Prof. Dr. med. Markus Hahn wird in offener Abstimmung mit einer Enthaltung gewählt

Dr. med. Siegfried Krishnabhakdi erklärt ebenfalls seine Bereitschaft zur erneuten Kandidatur als Beisitzer - ebenfalls kein weiter Vorschlag.

Ergebnis: Dr. med. Siegfried Krishnabhakdi wird in offener Abstimmung mit einer Enthaltung gewählt.

Alle Kandidaten nehmen die Wahl an. 
Ad TOP 9: Berichte aus den Sektionen und Arbeitskreisen

K.-S. Heling erläutert, dass die Sektionen und Arbeitskreisen im Rahmen der Erweiterten Vorstandssitzung ihre relevanten Punkte vorgestellt haben und fragt nach weiteren Punkten, die noch vorgestellt werden sollen.
Die Jahresberichte müssen bis März 2017 in der Geschäftsstelle eingereicht werden.

Keine weiteren Wortmeldungen

\section{Ad TOP 10: Verschiedenes}

K.-S. Heling bedankt sich bei A. Hagendorff für den gelungenen Kongress, bei allen Anwesenden für die Teilnahme und lädt die Mit- glieder der DEGUM zum nächsten DLT 2017 nach Linz/Österreich ein (11.-13.10.17)

Keine weiteren Wortmeldungen

Der Präsident beendet die Sitzung um 19.10 Uhr.

PD Dr. med. K.-S. Heling, Präsident Prof. Dr. A. Hagendorff, Sekretär 